\title{
BMJ Open The silent epidemic of obesity in The Gambia: evidence from a nationwide, population-based, cross-sectional health examination survey
}

$\overline{\text { Bai Cham (D) , }, 2,3 \text { Shaun Scholes, }{ }^{3} \text { Linda Ng Fat, }{ }^{3} \text { Omar Badjie, }{ }^{4} \text { Nora Ellen Groce, }{ }^{3}}$ Jennifer S Mindell (D) ${ }^{3}$

To cite: Cham B, Scholes S, $\mathrm{Ng}$ Fat $\mathrm{L}$, et al. The silent epidemic of obesity in The Gambia: evidence from a nationwide, populationbased, cross-sectional health examination survey. BMJ Open 2020;10:e033882. doi:10.1136/ bmjopen-2019-033882

- Prepublication history and additional material for this paper are available online. To view these files, please visit the journal online (http://dx.doi org/10.1136/bmjopen-2019033882).

Received 28 August 2019 Revised 28 January 2020 Accepted 23 April 2020

Check for updates

(c) Author(s) (or their employer(s)) 2020. Re-use permitted under CC BY-NC. No commercial re-use. See rights and permissions. Published by BMJ.

${ }^{1}$ Medical Research Council Unit The Gambia at the London School of Hygiene and Tropical Medicine, Banjul, The Gambia ${ }^{2}$ Department of Public Health, University of The Gambia, Brikama, The Gambia

${ }^{3}$ Research Department of Epidemiology and Public Health, University College London, London, UK

${ }^{4}$ Non-communicable Diseases Control Unit, Ministry of Health Banjul, The Gambia

Correspondence to

Dr Bai Cham;

barhamcham@hotmail.com

\section{ABSTRACT}

Objectives Non-communicable diseases account for $70 \%$ of global deaths; $80 \%$ occur in low-income and middleincome countries. The rapid increase of obesity in subSaharan Africa is a concern. We assessed generalised and abdominal obesity and their associated risk factors among adults in The Gambia.

Design Nationwide cross-sectional health examination survey using the WHO STEPwise survey methods.

Setting The Gambia.

Participants This study uses secondary analysis of a 2010 nationally representative random sample of adults aged 25-64 years (78\% response rate). The target sample size was 5280 , and 4111 responded. Analysis was restricted to non-pregnant participants with valid weight and height measurements $(n=3533)$.

Primary and secondary outcome measures The primary outcome variable was generalised obesity, using WHO body mass index (BMI) thresholds. Analyses used non-response weighting and adjusted for the complex survey design. We conducted multinomial logistic regression analysis to identify factors associated with BMI categories. The secondary outcome variable was abdominal obesity, defined as high waist circumference (using the International Diabetes Federation thresholds for Europeans).

Results Two-fifths of adults were overweight/obese, with a higher obesity prevalence in women $(17 \%, 95 \% \mathrm{Cl}$ 14.7 to 19.7 ; men $8 \%, 95 \%$ Cl 6.0 to 11.0 ). $10 \%$ of men and $8 \%$ of women were underweight. Urban residence (adjusted relative risk ratio $5.8,95 \% \mathrm{Cl} 2.4$ to 14.5 ), higher education (2.3, 1.2 to 4.5$)$, older age, ethnicity, and low fruit and vegetable intake $(2.8,1.1$ to 6.8$)$ were strongly associated with obesity among men. Urban residence $(4.7,2.7$ to 8.2$)$, higher education $(2.6,1.1$ to $6.4)$, older age and ethnicity were associated with obesity in women.

Conclusion There is a high burden of overweight/ obesity in The Gambia. While obesity rates in rural areas were lower than in urban areas, obesity prevalence was higher among rural residents in this study compared with previous findings. Preventive strategies should be directed at raising awareness, discouraging harmful beliefs on weight, and promoting healthy diets and physical activity.
Strengths and limitations of this study

- Our study uses the most recent nationally representative data on generalised and abdominal obesity among adults in The Gambia, and hence it serves as a baseline study from which future changes in prevalence and risk factors can be assessed.

- The complex sampling strategy and the stringent WHO STEP protocols applied in collecting the data, particularly the use of objective anthropometric measurements taken by trained field staff, minimised biases.

- The study has identified population subgroups to prioritise with health promotion measures.

- Our main limitation is that the survey did not collect self-reported measures on beliefs about body size and weight management, which are important in The Gambian context to assess and monitor trends on beliefs and practices.

- We also had only one complete measure of socioeconomic position (education), as missing information on income was high.

\section{INTRODUCTION}

Non-communicable diseases (NCDs) are increasing in sub-Saharan Africa (SSA), ${ }^{2}$ and account for $71 \%$ of all deaths globally. They also account for 15 million premature deaths among adults aged 30-69 years; $85 \%$ of these premature deaths occur in low-income and middle-income countries. ${ }^{2}$ A pooled analysis of 1698 population-based measurement studies comprising 19 million participants from 200 countries revealed an increasing trend of obesity globally. ${ }^{3}$ If these trends continue, meeting the WHO global NCD target of halting the rise of obesity by 2025 is almost impossible. ${ }^{4}$

A great concern is the rapid increase of obesity in SSA. ${ }^{1}$ Countries in SSA face the challenge of the double burden of communicable and non-communicable diseases, and also the double burden of underweight/malnutrition 
and obesity. ${ }^{5-7}$ A pooled analysis of population-based studies from 1980 to 2014 in Africa demonstrated a significant increase in age-standardised mean body mass index (BMI) across the continent. ${ }^{8} \mathrm{~A}$ recent analysis of Demographic and Health Surveys conducted between 1991 and 2014 in 24 African countries revealed a significant increase in obesity among women; rates in some countries tripled. ${ }^{9}$ There is evidence suggesting obesity is increasing more quickly in developing countries, especially in SSA, compared with developed countries. ${ }^{1011}$ This is associated with a range of factors, including epidemiological and nutritional transition, adoption of Western lifestyles, decreased physical activity, low fruit and vegetable consumption, increased consumption of processed foods, and urbanisation. ${ }^{12-15}$

Few studies on obesity have been conducted in The Gambia and most of them are either not nationally representative or are out of date. A study using data from 1942 to 1997 on the causes of death in The Gambian capital Banjul documented the double burden of noncommunicable diseases with communicable diseases exacerbated by malnutrition. ${ }^{16}$ In a nationwide assessment among Gambians aged 16 years and above in 1996, $18 \%$ were underweight, $8 \%$ overweight and $2 \%$ obese. $^{17}$ A related study in urban and rural communities in The Gambia revealed that $18 \%$ of participants were underweight and $4 \%$ were obese, with a higher prevalence of obesity (33\%) among urban women aged 35 years and above. ${ }^{18}$ Both studies confirmed the persistence of the double burden of underweight and overweight in The Gambia, although obesity prevalence was low (but increasing) in those surveys.

The double burden of communicable and noncommunicable diseases poses a challenge to governments and families in SSA; The Gambia is no exception. We recently demonstrated a high prevalence of hypertension in The Gambia, with a greater burden in rural areas and among adults classified as obese. ${ }^{19}$ There is also a high prevalence of smoking among Gambian men. ${ }^{20}$ Moreover, these health risks have significant implications for wider development concerns. It poses a barrier to poverty alleviation and can hinder the attainment of the United Nations Sustainable Development Goals, particularly target 3.4, which calls for a reduction in premature mortality due to NCDs by one-third by $2030 .{ }^{21}{ }^{22}$ Halting the rise of obesity is also one of the WHO 2025 targets for the reduction of NCD mortality. ${ }^{4}$ Using the most recent nationally representative data, including objective anthropometric measurements, the aim of this study was to assess the burden of underweight, overweight and obesity among adults (aged 25-64 years) in The Gambia.

\section{METHODS}

\section{Participants, sampling strategy and data collection}

Our study is based on secondary analysis of data from the most recent nationally representative, population-based health examination survey conducted in The Gambia.
The study setting and design, sampling, and research instruments have been previously described. ${ }^{19}{ }^{20}$ Briefly, data were collected from a random sample of adults aged 25-64 years from January to March 2010 using the WHO STEPwise approach. ${ }^{19} 23$ Participants were selected using a multistage stratified sampling technique based on the 2003 population census of The Gambia. The country's eight local government areas served as strata for the sampling; 264 of the 408 enumeration areas (EAs) were then selected across the country and 20 households selected from each EA, both stages by simple random sampling. One eligible participant aged 25-64 years was sampled from each selected household, using the Kish method. Sampled participants who were not reached after three or more visits and those who declined were not replaced. The target sample was set at 5280; 4111 responded (response rate $78 \%$ ). Because of the complex sampling design, sample weights and poststratification weights were applied to account for differences in the selection probability and to adjust for differences between the national age-sex distribution and that of the achieved sample.

The anthropometric measurements were performed by fieldworkers at the participant's residence. Weight, height and waist circumference were measured using WHO STEP protocols. ${ }^{23}$ The measurements were conducted using standard scales with participants wearing light clothing, with footwear and headwear removed. Weight was measured to the nearest $0.1 \mathrm{~kg}$ using digital bathroom scales. Height was measured to the nearest $0.1 \mathrm{~cm}$ in standing position using standard portable stadiometers. Waist circumference was measured (once) to the nearest $0.1 \mathrm{~cm}$ using a tape measure and was taken midway between the lowest rib and the iliac crest.

\section{Dependent/outcome variables}

The first outcome variable was generalised obesity, defined using BMI calculated by dividing weight (in $\mathrm{kg}$ ) by height squared $\left(\mathrm{m}^{2}\right)$. We categorised BMI into underweight (BMI $<18.5 \mathrm{~kg} / \mathrm{m}^{2}$ ), normal/desirable weight $\left(18.5-24.9 \mathrm{~kg} / \mathrm{m}^{2}\right)$, overweight $\left(25.0-29.9 \mathrm{~kg} / \mathrm{m}^{2}\right)$ and obese (BMI $\left.\geq 30 \mathrm{~kg} / \mathrm{m}^{2}\right)$, using the WHO thresholds. ${ }^{24} \mathrm{We}$ used abdominal obesity (high waist circumference) as the second outcome variable, defined using the International Diabetes Federation thresholds ( $\geq 90 \mathrm{~cm}$ in men and $\geq 80$ $\mathrm{cm}$ in women). ${ }^{25}$

\section{Independent covariates/predictor variables}

The predictor variables included sociodemographic and behavioural risk factors including self-reported age group, ethnicity, education (years of education: $\leq 6,7-12,>12$ ), residence, fruit and vegetable intake, physical inactivity, and smoking (categories shown in online supplementary table S1). There was a high amount of missing information on income and hence we used level of education as a measure of socioeconomic position. 


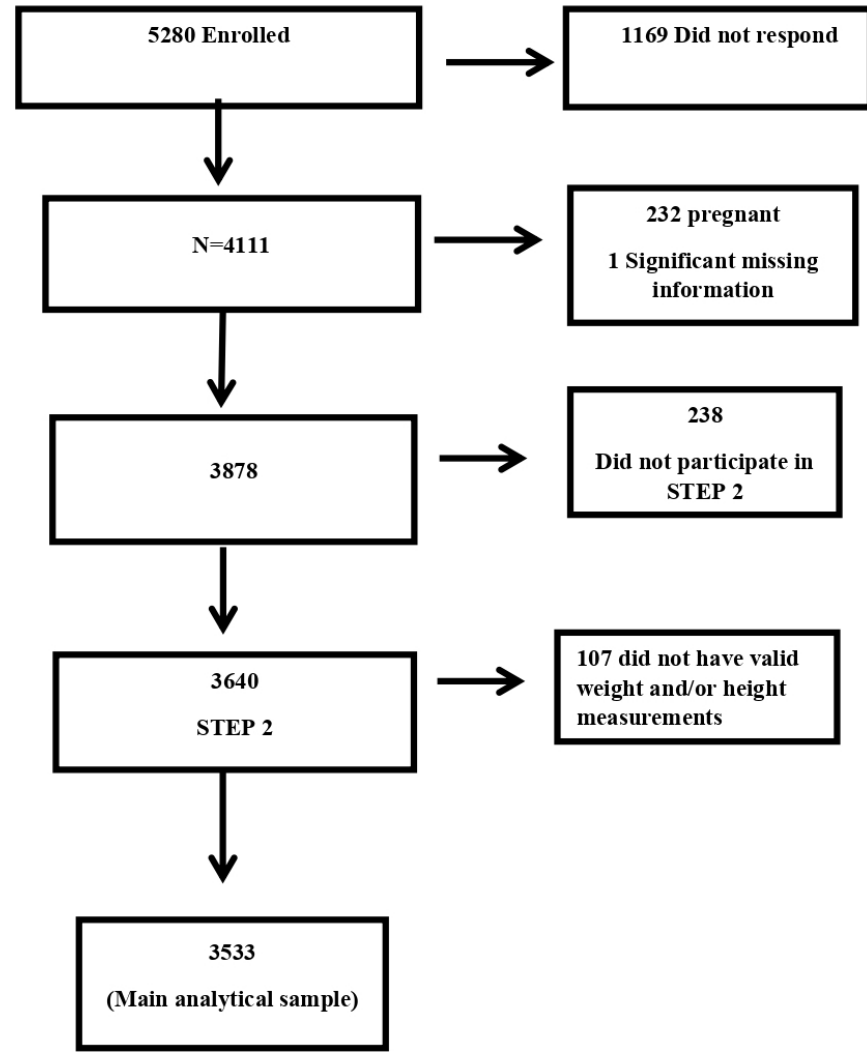

Figure 1 Flow diagram of study participants with number excluded and reason for exclusion.

\section{Statistical analysis}

The analytical sample was restricted to non-pregnant participants with valid weight and height data $(\mathrm{n}=3533)$. Figure 1 outlines the number of participants sampled, the number excluded due to specific reasons and the number included in the final analysis.

Complete case analysis was performed as fewer than $1 \%$ of adults with valid weight and height had missing information on other variables. In descriptive analyses, we summarised participants' sociodemographic characteristics as well as their behavioural risk factors.

The prevalence of BMI categories is reported as proportions with their corresponding 95\% CIs. We conducted multivariable multinomial logistic regression analysis to identify factors associated with being underweight, overweight and obese separately, comparing each of these categories with the reference category of normal/desirable weight. Sociodemographic and behavioural risk factors in the data set that are known or thought to be associated with obesity were included. We excluded smoking (in women) and alcohol consumption (both sexes) from the regression models due to their low prevalence. However, model fit or adequacy was not assessed. Age-adjusted and fully adjusted relative risk ratios (ARRR), with their corresponding 95\% CIs, are reported. All analyses were stratified by gender, as we expected that the associations between the predictors and outcomes may differ by gender.
Due to the collinearity of the two variables on residence (ie, local government area and rurality), fully adjusted models were repeated interchanging these variables. We explored variables that could modify the association between BMI categories and the covariates by fitting interaction terms. There was no evidence of modification (all $\mathrm{p}>0.05$ ) and hence multinomial regression models without interaction terms are reported. As in other studies, we did not include abdominal obesity in the models for BMI because of the collinearity of waist circumference and BMI. ${ }^{26}$

We explored the factors associated with abdominal obesity (high waist circumference as defined above) by conducting multivariable binary logistic regression analysis. BMI was not included as a predictor in these models because of the aforementioned collinearity of waist circumference and BMI. For abdominal obesity, age-adjusted OR and fully adjusted OR (AOR) with corresponding 95\% CI are reported.

All analyses were weighted for non-response and adjusted for the complex survey design in accordance with WHO STEPwise protocols. Analyses were performed using Stata V.15.

\section{Patient and public involvement}

Patients and the public were not directly involved in this study. However, the STEPwise survey on which the data reported in this study are based was population-based. All the interviews and anthropometric measurements were conducted at the participant's residence. Prior to the survey, people were sensitised about the objectives of the survey and its importance through radio, television, community meeting places and so on. Results from the previous analyses have been shared. In addition, the results are used by the Ministry of Health of The Gambia in their routine sensitisation campaigns. Like our previous analyses ${ }^{19}{ }^{20}$ the results of this study will be shared with the public and will also be used to inform policy.

\section{RESULTS}

\section{Characteristics of participants}

Descriptions of respondents' sociodemographic, behavioural risk factors and anthropometry data are presented in online supplementary table S1. The unadjusted mean age was $38.3 \pm 10.9$ years. More than two-fifths $(44 \%)$ of the participants were in the youngest age group (25-34 years), particularly among women (53\% vs $33 \%$ of men). However, there was no age difference by gender after weighting and adjusting for the complex survey design $(p=0.937$; online supplementary table $\mathrm{S} 1)$. The adjusted mean BMI was $24.6 \mathrm{~kg} / \mathrm{m}^{2}$ (95\% CI 24.1 to 25.1) and the mean waist circumference was $74.0 \mathrm{~cm}(71.1-$ 76.9). The mean BMI and waist circumference were both higher among women: BMI $23.6 \mathrm{~kg} / \mathrm{m}^{2}(23.1-24.1 \mathrm{~kg} /$ $\left.\mathrm{m}^{2}\right)$ in men vs $25.6 \mathrm{~kg} / \mathrm{m}^{2}\left(24.9-26.3 \mathrm{~kg} / \mathrm{m}^{2}\right)$ in women, and waist circumference $72.1 \mathrm{~cm}(65.1-75.0 \mathrm{~cm})$ in men compared with $76.0 \mathrm{~cm}(72.9-79.1 \mathrm{~cm})$ in women. 


\section{Prevalence of underweight, overweight and obesity}

The prevalence of BMI categories by selected sociodemographic and behavioural characteristics is presented for men and women in online supplementary tables S2 and S3, respectively. Among men, more than half had a normal/desirable weight (56\%, 95\% CI 50.8\% to $61.4 \%$ ) and one in ten was underweight $(10 \%, 95 \%$ CI $7.6 \%$ to $12.4 \%)$. The prevalence of overweight and obesity in men was $26 \%(21.1 \%-31.6 \%)$ and $8 \%(6.0 \%-11.0 \%)$, respectively (online supplementary table S2). Almost half of women were either overweight $(29 \%, 25.8 \%-31.9 \%)$ or obese $(17 \%, 14.7 \%-19.7 \%)$, while $8 \%(6.1 \%-9.5 \%)$ were underweight (online supplementary table S3). Among both men and women, the prevalence of overweight and of obesity was substantially higher among urban residents, those with a higher level of education and those physically inactive. More than $60 \%$ of the residents in the capital (Banjul) and the nearby towns (Kanifing Municipality) were either overweight or obese. Obesity was also high among never smokers and ex-smokers in men. The prevalence of abdominal obesity was 10\% (95\% CI 7.8\% to $13.4 \%$ ) in men and $46 \%$ (95\% CI $39.3 \%$ to $52.6 \%$ ) in women (data not shown).

\section{Factors associated with underweight, overweight and obesity}

Factors strongly associated with generalised obesity (vs normal/desirable weight) in the multivariable multinomial logistic regressions included older age, ethnicity, higher education and urban residence among both men and women (tables 1 and 2). Obesity was also associated with low fruit and vegetable consumption (ARRR 2.8, 95\% CI 1.1 to 6.8 ) in men. All these variables with the exception of ethnicity in men were also strongly associated with overweight (vs normal weight), while current smoking was inversely associated with overweight $(0.5$, 0.4-0.7). Compared with rural residents, the associations of overweight and obesity among urban residents were threefold and sixfold higher, respectively, in men (overweight $2.8,1.5-5.0$; obesity $5.8,2.4-14.5$ ) and threefold and fivefold higher in women (overweight 3.1, 1.9-5.0; obesity 4.7, 2.7-8.2). Physical inactivity was strongly associated with obesity among both men and women in the age-adjusted models but not in the fully adjusted models, although the direction of the association remained unchanged (tables 1 and 2).

No strong associations were found for underweight (vs normal/desirable weight) in men except for an increased ARRR among ex-smokers (ARRR 1.9, 1.1-3.2) and an inverse association with being Fula $(0.5,0.2-0.9)$ or of minority ethnicity $(0.4,0.1-1.0)$ compared with being Mandinka (table 1). Among women, the risk of being underweight (vs normal weight) was higher among those aged 55-64 years compared with those aged 25-34 years (2.3, 95\% CI 1.1 to 4.8$)$ and was inversely related with semiurban residence compared with rural residence $(0.5,0.3-1.0)$ and with minority ethnicity compared with Mandinka (0.3, 0.1-0.8) (table 2).

\section{Factors associated with abdominal obesity}

In the fully adjusted multivariable binary logistic regression model, older age, residence, low fruit and vegetable intake (men only) and being an ex-smoker compared with never smoking (men only) were strongly associated with higher odds of abdominal obesity (table 3 ). Semiurban residence (AOR 0.4, 95\% CI 0.2 to 0.9 ) compared with rural residence, and low fruit and vegetable intake (0.6, 0.4-0.9) compared with the recommended intake of at least five servings a day, were inversely associated with the odds of abdominal obesity among men. Older age (3.2, 2.1-4.9) compared with younger age, and semiurban residence (2.1, 1.2-3.7) compared with rural residence, were associated with higher odds of abdominal obesity among women (table 3 ).

\section{DISCUSSION}

Using the most recent nationally representative data, including objective anthropometric measurements, the aim of this study was to assess the burden of underweight, overweight and obesity among adults (aged 25-64 years) in The Gambia. This study has shown that the burden of overweight and obesity is high in The Gambia, especially among women (29\% and $17 \%$, respectively) and urban residents. No precise quantification of changes over time in prevalence can be made since the only previous nationwide study was based on a different age cohort. ${ }^{17}$ Nevertheless, we can reasonably assume that the prevalence of obesity has increased substantially in The Gambia within a period of less than 15 years. Almost half of women and more than one-third of men aged 25-64 years were either overweight or obese in 2010, while the prevalence of overweight and obesity in 1996 was $8 \%$ and 2\%, respectively, among participants aged 16 years and above. The prevalence of underweight, however, halved from $18 \%$ in 1996 to $9 \%$ in this study. This shows an increasing shift from malnutrition/underweight to overweight and obesity among Gambian adults. These changes reflect shifts in growing economic progress, modernisation of household tasks, improved transportation and increasing urbanisation.

The prevalence of obesity in The Gambia is more than double the levels reported in similar national WHO STEPwise surveys conducted in Malawi, ${ }^{27}$ Eritrea $^{28}$ and Mozambique, ${ }^{29}{ }^{30}$ but is less than that reported in the Republic of Seychelles. ${ }^{31}$ The high prevalence of obesity in The Gambia is a cause for concern, given the increasing burden of NCDs, notably hypertension. ${ }^{19}$ Although higher in urban areas, generalised obesity is now a problem in both urban and rural areas in The Gambia, in contrast to the evidence from previous studies. ${ }^{1718}$ Despite the health risks associated with overweight/obesity, Gambians are culturally obesity-tolerant. ${ }^{32} 33$ It has been well documented that perceptions of body weight vary across different parts of the world. ${ }^{34} 35$ In some parts of SSA, being overweight is not perceived as a risk factor for NCDs but rather as a sign of beauty, wealth, success and prestige; 


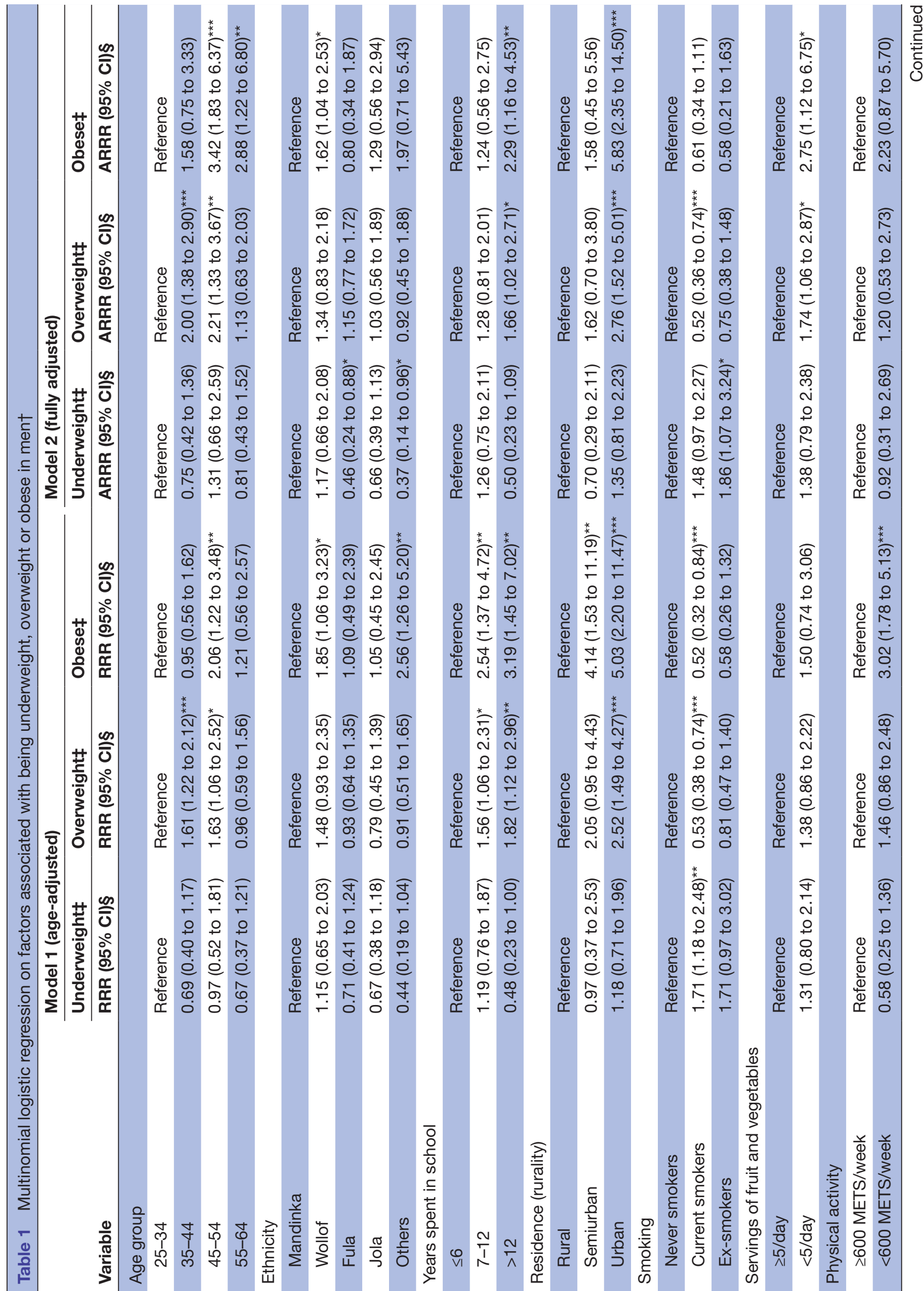

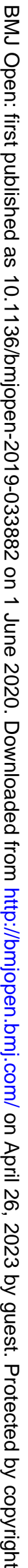




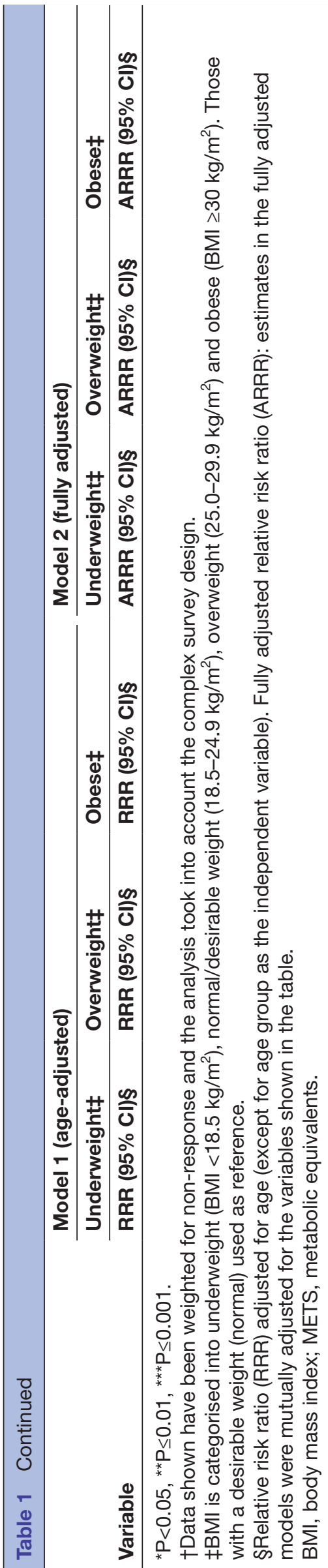

such cultural beliefs encourage obesity. ${ }^{34}$ This is the case in The Gambia; a study on the perception of body image and attractiveness among adults in urban areas in The Gambia demonstrated high satisfaction with big body image (overweight), especially among women. ${ }^{32} \mathrm{~A}$ cross-cultural comparison using published data on Figure Rating Scales found that Gambians' rating of a 'normal' weight was bigger than those of North Americans, and that Gambians were more tolerant of obesity than white and African-Americans. ${ }^{32}$ A related study also conducted in The Gambia showed that weight gain was not associated with weight concern, as $68 \%$ of those overweight and $37 \%$ of those obese did not perceive themselves to be overweight/obese. ${ }^{33}$ Findings from other SSA countries have indicated that women tend to frame fatness as a symbol of wealth, as has been found, for example, in Senegal ${ }^{36} 37$ and in Zambia. ${ }^{38}$ Associating overweight/ obesity with beauty and prestige/wealth renders the burden of obesity a silent epidemic, as many people in The Gambia do not consider it a risk or want to address it.

Our models showed that older age, ethnicity, higher education and urban residence in both genders, and low fruit and vegetable intake and smoking in men, were strongly associated with the risks of overweight and obesity (vs normal/desirable weight). Evidence links urbanisation and the increasing burden of obesity and other NCDs, especially in low-income countries. ${ }^{39-42}$ Higher education was also significantly associated with overweight and obesity in our study. Highly educated adults in The Gambia are more likely to be in office jobs, which are mostly sedentary. Physical inactivity was strongly associated with obesity in the age-adjusted regression models among both men and women. However, this relationship failed to attain statistical significance after full adjustment for social and demographic factors, suggesting that social and demographic factors may be confounding the age-adjusted relationship between physical inactivity and obesity. Leisure time physical activity was low among the study participants; only $12 \%$ of adults in the present study reported engaging in any form of leisure time activity: most of the physical activity reported was therefore work-related and transport-related. Judging from the data, participants with a higher level of education therefore had lower levels of physical activity and hence were more prone to obesity. There is evidence suggesting that increases in the level of physical activity and/or involvement in exercise interventions-whether supervised or not-have a positive impact on BMI and overall health. ${ }^{43}$ Given our evidence that leisure time physical activity is low in The Gambia, the Ministry of Health and Social Welfare of The Gambia and its stakeholders should promote physical activity at the individual and population levels. As the promotion of physical activity, especially at the population level, is multidisciplinary, it should be done in collaboration with other government line ministries, municipalities, community-based organisations and non-governmental organisations. The goal of the recent WHO Global Action Plan on Physical Activity 


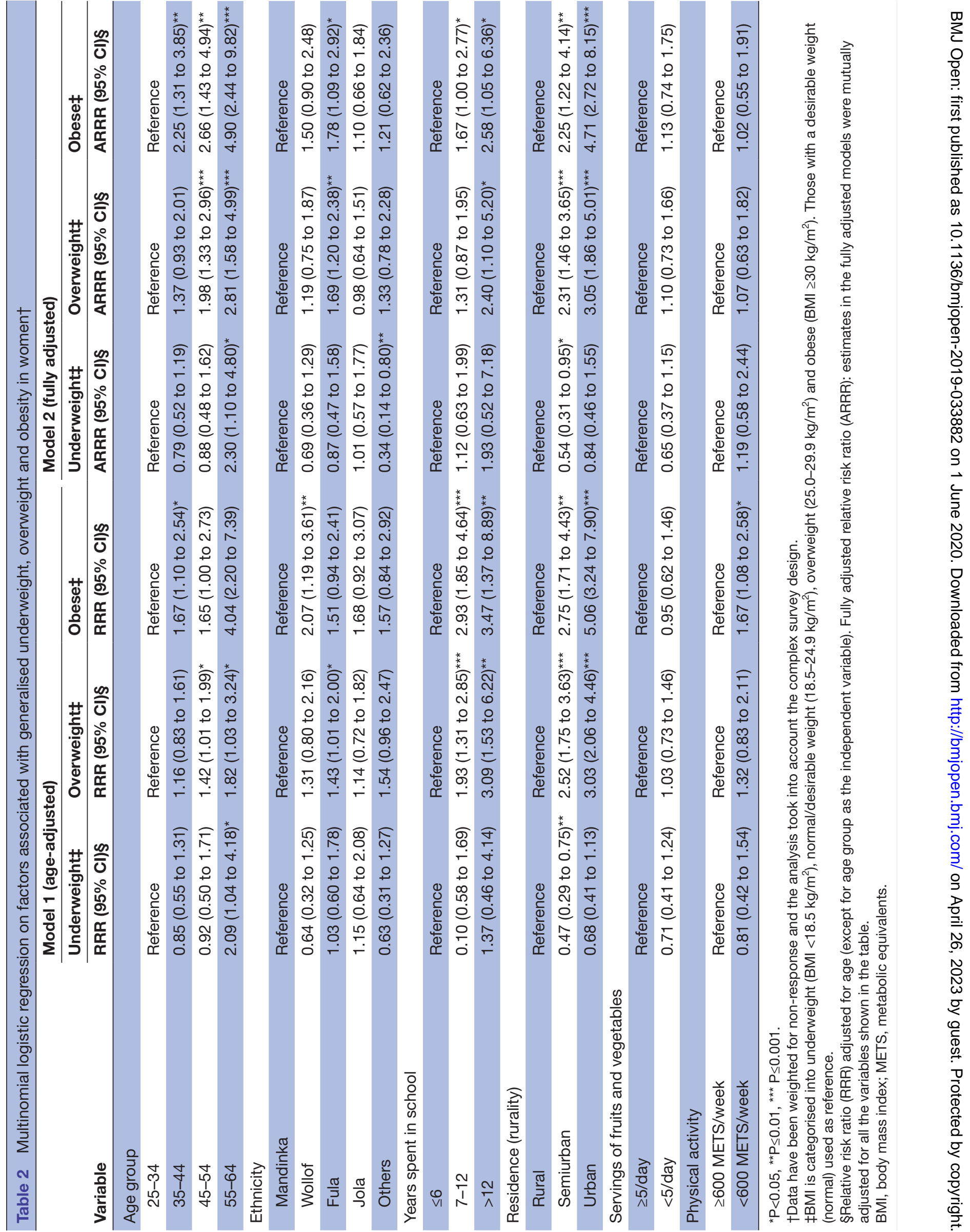




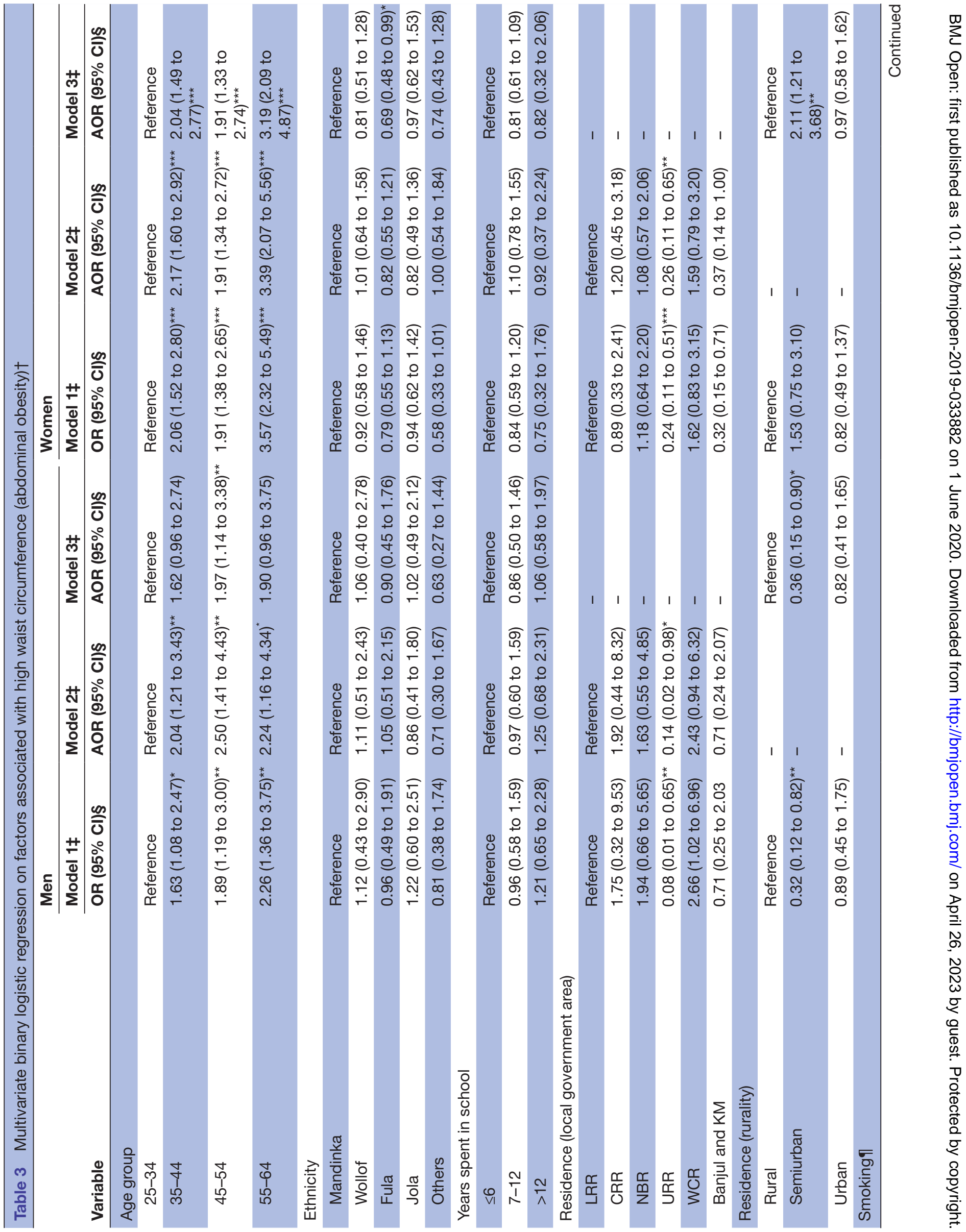




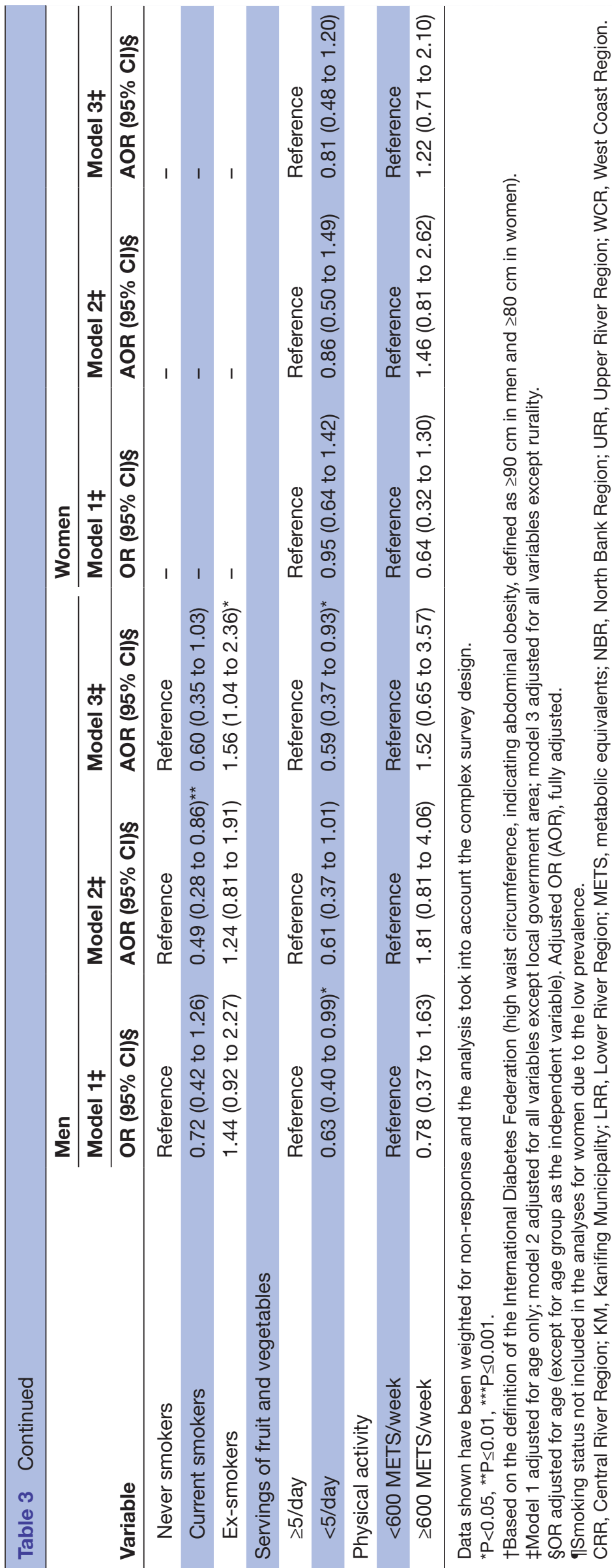

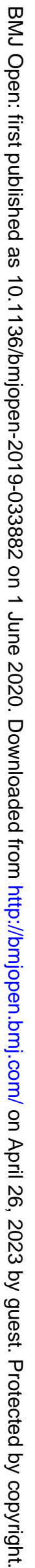


2018-2030 ('more active people for a healthier world') is to reduce the global prevalence of physical inactivity by $15 \%$ by $2030 .{ }^{44}$ Our findings support the advisability of the Ministry of Health of The Gambia incorporating this in its national health policy and/or the NCDs policy and strategic plan.

Low fruit and vegetable intake (defined as having fewer than five combined servings a day) was associated with obesity in our study, especially among men. There is a strong linkage between low fruit and vegetable consumption and increased NCD risk. Regular consumption of fruits and vegetables may help prevent unhealthy weight gain, especially when taken as part of a healthy diet. ${ }^{45} \mathrm{~A}$ systematic analysis for the Global Burden of Disease Study in 2010 attributed more than six million deaths globally to inadequate consumption of fruits and vegetables. ${ }^{47} \mathrm{An}$ additional finding from our data was that the consumption of fruits and vegetables was low; consumption of fruits and vegetables as part of healthy diets should therefore be widely promoted. Future health examination surveys to monitor indicators such as overweight/obesity in The Gambia should include a more comprehensive assessment of diet (including unhealthy or fast food consumption) than that collected in the 2010 survey.

Being an ex-smoker in men and older age in women were positively associated with being underweight (vs normal weight) in the fully adjusted analyses presented here. Semiurban residents were less likely to be underweight (vs normal weight) compared with rural residents. The association of underweight with being an ex-smoker might be at least partly explained by the associations of both with ill health. It is possible that ex-smokers were advised to quit smoking because of their illness. Moreover, the association of underweight with older age in women could also be associated with age-related illnesses. Poverty, especially in rural areas, may explain the inverse association of underweight with semiurban compared with rural residence among women.

A potential positive finding from this study is that higher rates of obesity were found among those with higher education and more urban-based members of the population, the very people who may be most effectively reached by public health campaigns.

\section{Strengths and limitations of this study}

This study presents the most recent nationally representative data on obesity among adults in The Gambia. It gives a better picture of the true burden of obesity in the country and hence could serve as a baseline study from which future changes can be assessed. The complex sampling strategy and the stringent WHO STEP protocols applied in collecting the data, particularly the use of objective measurements taken by trained field staff instead of a reliance on self-reported anthropometric data, minimised biases.

Our main limitation is the cross-sectional nature of the study, which prevents attribution of causality to the associations. However, it does identify population subgroups to prioritise with health promotion measures. There is a possibility of misclassifying obesity in people who are physically active and have large muscle mass. For this reason we explored abdominal obesity as an additional outcome variable. Of the participants who took part in the physical measurements, $3 \%$ did not have valid weight and height measurements, which could have led to nonresponse bias. However, we compared the two groups and there were no systematic differences between those with and without valid anthropometric measurements (data not shown). We had only one complete measure of socioeconomic position (education) as missing information on household income was high, a common finding in surveys. Therefore, we were unable to estimate the associations between education and the outcome variables after adjustment for income. Our findings could have been influenced by this, and other unmeasured confounders such as fast food intake.

Currently, there is no standard threshold for high waist circumference in SSA, but the International Diabetes Federation recommends using the thresholds for Europeans ( $\geq 94 \mathrm{~cm}$ in men; $\geq 80 \mathrm{~cm}$ in women) for adults in SSA. ${ }^{25}$ However, a study that used data from different countries as part of the African Partnership for Chronic Disease Research revealed optimal waist circumference cut-off point for identifying men at increased cardiometabolic risk is lower $(\geq 81.2 \mathrm{~cm})$ than current guidelines for men in SSA, and similar to that of women. ${ }^{48}$ We therefore used the International Diabetes Federation thresholds for Asians ( $\geq 90 \mathrm{~cm}$ in men; $\geq 80 \mathrm{~cm}$ in women). ${ }^{25}$ The data shown on waist circumference levels may therefore be underestimated or overestimated compared with alternative thresholds for abdominal obesity. Finally, the survey did not collect information on beliefs about body size and weight management, which are important in The Gambian context to assess and monitor trends on beliefs and practices.

\section{CONCLUSION}

This study reveals a high prevalence of obesity among Gambian adults, while the burden of underweight in this population may be decreasing. There are likely to be sociocultural norms that promote overweight, especially among women. Preventive strategies should be directed at raising awareness of the importance of achieving and maintaining a healthy weight, discouraging harmful sociocultural practices and beliefs about weight, and promoting healthy diets and regular physical activity during leisure time, particularly in urban areas and among women.

\section{Twitter Jennifer S Mindell @j_mindell}

Acknowledgements We thank the WHO for the technical and financial support to conduct the survey and the Ministry of Health of The Gambia for the authorisation to use the data.

Contributors BC conceptualised the paper, analysed the data and wrote the first draft of the manuscript. JSM, SS, NEG and LNF revised the work critically for 
important academic content. OB supervised the survey data collection process and contributed to the revision of the manuscript. All the authors approve the final version of the manuscript.

Funding $\mathrm{BC}$ 's PhD research at UCL (University College London) was funded by the Islamic Development Bank.

Competing interests None declared.

Patient and public involvement Patients and/or the public were involved in the design, or conduct, or reporting, or dissemination plans of this research. Refer to the Methods section for further details.

Patient consent for publication Not required.

Ethics approval Ethical approval for the survey was obtained from the National Ethics Committee of The Gambia; participants gave verbal or written consent.

Provenance and peer review Not commissioned; externally peer reviewed.

Data availability statement The Gambia 2010 WHO STEP data are not publicly available but can be obtained from a third party upon request.

Open access This is an open access article distributed in accordance with the Creative Commons Attribution Non Commercial (CC BY-NC 4.0) license, which permits others to distribute, remix, adapt, build upon this work non-commercially, and license their derivative works on different terms, provided the original work is properly cited, appropriate credit is given, any changes made indicated, and the use is non-commercial. See: http://creativecommons.org/licenses/by-nc/4.0/.

\section{ORCID iDs}

Bai Cham http://orcid.org/0000-0002-1656-2126

Jennifer S Mindell http://orcid.org/0000-0002-7604-6131

\section{REFERENCES}

1 Dalal S, Beunza JJ, Volmink J, et al. Non-Communicable diseases in sub-Saharan Africa: what we know now. Int J Epidemiol 2011;40:885-901.

2 WHO. Noncommunicable diseases Factsheet, 2019. Available: http:// www.who.int/mediacentre/factsheets/fs355/en/ [Accessed 10 Jan 2020]

3 NCD Risk Factor Collaboration (NCD-RisC). Trends in adult bodymass index in 200 countries from 1975 to 2014: a pooled analysis of 1698 population-based measurement studies with $19 \cdot 2$ million participants. Lancet 2016;387:1377-96.

4 WHO. Global action plan for the prevention and control of noncommunicable diseases 2013-2020, 2013.

5 Boutayeb A. The double burden of communicable and noncommunicable diseases in developing countries. Trans $R$ Soc Trop Med Hyg 2006;100:191-9.

6 Nyirenda MJ. Non-Communicable diseases in sub-Saharan Africa: understanding the drivers of the epidemic to inform intervention strategies. Int Health 2016;8:157-8.

7 Popkin BM, Corvalan C, Grummer-Strawn LM. Dynamics of the double burden of malnutrition and the changing nutrition reality. Lancet 2020;395:65-74.

8 NCD Risk Factor Collaboration (NCD-RisC) - Africa Working Group. Trends in obesity and diabetes across Africa from 1980 to 2014: an analysis of pooled population-based studies. Int J Epidemiol 2017;46:1421-32.

9 Amugsi DA, Dimbuene ZT, Mberu B, et al. Prevalence and time trends in overweight and obesity among urban women: an analysis of demographic and health surveys data from 24 African countries, 1991-2014. BMJ Open 2017;7:e017344.

10 Popkin BM, Slining MM. New dynamics in global obesity facing lowand middle-income countries. Obes Rev 2013;14 Suppl 2:11-20.

11 Owolabi EO, Ter Goon D, Adeniyi OV. Central obesity and normalweight central obesity among adults attending healthcare facilities in buffalo City metropolitan Municipality, South Africa: a cross-sectional study. J Health Popul Nutr 2017;36:54.

12 Ojofeitimi EO, Adeyeye AO, Fadiora AO, et al. Awareness of obesity and its health hazard among women in a university community. Pakistan J Nutr 2007;6:502-5.

13 Doku DT, Neupane S. Double burden of malnutrition: increasing overweight and obesity and stall underweight trends among Ghanaian women. BMC Public Health 2015;15:670.

14 Biadgilign S, Mgutshini T, Haile D, et al. Epidemiology of obesity and overweight in sub-Saharan Africa: a protocol for a systematic review and meta-analysis. BMJ Open 2017;7:e017666.
15 Bosu WK. An overview of the nutrition transition in West Africa: implications for non-communicable diseases. Proc Nutr Soc 2015;74:466-77.

16 van der Sande MA, Inskip HM, Jaiteh KO, et al. Changing causes of death in the West African town of Banjul, 1942-97. Bull World Health Organ 2001;79:133-41.

17 van der Sande MA, Bailey R, Faal H, et al. Nationwide prevalence study of hypertension and related non-communicable diseases in the Gambia. Trop Med Int Health 1997;2:1039-48.

18 van der Sande MA, Ceesay SM, Milligan PJ, et al. Obesity and undernutrition and cardiovascular risk factors in rural and urban Gambian communities. Am J Public Health 2001;91:1641-4.

19 Cham B, Scholes S, Ng Fat L, et al. Burden of hypertension in The Gambia: evidence from a national World Health Organization (WHO) STEP survey. Int J Epidemiol 2018;47:860-71.

20 Cham B, Scholes S, Groce NE, et al. Prevalence and predictors of smoking among Gambian men: a cross-sectional national WHO STEP survey. Int J Environ Res Public Health 2019;16:4719.

21 Clark H. NCDs: a challenge to sustainable human development. Lancet 2013;381:510-1.

22 Lal P, Jacob A, Buragohain A. UN development programme and noncommunicable diseases. Lancet 2013;382:305.

23 WHO. STEPS: a framework for surveillance: the WHO stepwise approach to surveillance of non-communicable diseases steps, 2003.

24 WHO. Obesity: preventing and managing the global epidemic. Report of a WHO consultation. World Health Organ Tech Rep Ser 2000;894:1-253.

25 International Diabetes Federation. The IDF consensus worldwide definition of the metabolic syndrome. Brussels, 2006.

26 Han TS, van Leer EM, Seidell JC, et al. Waist circumference action levels in the identification of cardiovascular risk factors: prevalence study in a random sample. BMJ 1995;311:1401-5.

27 Msyamboza KP, Kathyola D, Dzowela T. Anthropometric measurements and prevalence of underweight, overweight and obesity in adult Malawians: nationwide population based NCD STEPS survey. Pan Afr Med J 2013;15:108.

28 Mufunda J, Mebrahtu G, Usman A, et al. The prevalence of hypertension and its relationship with obesity: results from a national blood pressure survey in Eritrea. J Hum Hypertens 2006;20:59-65.

29 Gomes A, Damasceno A, Azevedo A, et al. Body mass index and waist circumference in Mozambique: urban/rural gap during epidemiological transition. Obes Rev 2010;11:627-34.

30 Damasceno A, Azevedo A, Silva-Matos C, et al. Hypertension prevalence, awareness, treatment, and control in Mozambique: urban/rural gap during epidemiological transition. Hypertension 2009;54:77-83.

31 Faeh D, William J, Tappy L, et al. Prevalence, awareness and control of diabetes in the Seychelles and relationship with excess body weight. BMC Public Health 2007;7:163.

32 Siervo M, Grey P, Nyan OA, et al. A pilot study on body image, attractiveness and body size in Gambians living in an urban community. Eat Weight Disord 2006;11:100-9.

33 Siervo M, Grey P, Nyan OA, et al. Urbanization and obesity in the Gambia: a country in the early stages of the demographic transition. Eur J Clin Nutr 2006;60:455-63.

34 Gele AA, Mbalilaki AJ. Overweight and obesity among African immigrants in Oslo. BMC Res Notes 2013;6:119.

35 Scott A, Ejikeme CS, Clottey EN, et al. Obesity in sub-Saharan Africa: development of an ecological theoretical framework. Health Promot Int 2013;28:das038:4-16.

36 Holdsworth M, Gartner A, Landais E, et al. Perceptions of healthy and desirable body size in urban Senegalese women. Int J Obes Relat Metab Disord 2004;28:1561-8.

37 Macia E, Cohen E, Gueye L, et al. Prevalence of obesity and body size perceptions in urban and rural Senegal: new insight on the epidemiological transition in West Africa. Cardiovasc J Afr 2017;28:324-30.

38 Tateyama Y, Musumari PM, Techasrivichien T, et al. Dietary habits, body image, and health service access related to cardiovascular diseases in rural Zambia: a qualitative study. PLoS One 2019;14:e0212739.

39 Godfrey R, Julien M. Urbanisation and health. Clin Med 2005;5:137-41.

40 Kruger HS, Venter CS, Vorster HH. Obesity in African women in the North West Province, South Africa is associated with an increased risk of non-communicable diseases: the THUSA study. transition and health during urbanisation of South Africans. Br J Nutr 2001;86:733-40.

41 Ojiambo RM. Are lifestyle shifts fuelling the obesity epidemic in urbanised Africans? Glob Health Promot 2016;23:73-5. 
42 Vorster $\mathrm{HH}$. The emergence of cardiovascular disease during urbanisation of Africans. Public Health Nutr 2002;5:239-43.

43 Ruotsalainen H, Kyngäs H, Tammelin T, et al. Systematic review of physical activity and exercise interventions on body mass indices, subsequent physical activity and psychological symptoms in overweight and obese adolescents. J Adv Nurs 2015;71:2461-77.

44 WHO. Global action plan on physical activity 2018-2030: more active people for a healthier world, 2018.

45 Alinia S, Hels O, Tetens I. The potential association between fruit intake and body weight--a review. Obes Rev 2009;10:639-47.
46 Ledoux TA, Hingle MD, Baranowski T. Relationship of fruit and vegetable intake with adiposity: a systematic review. Obes Rev 2011;12:e143-50.

47 Lim SS, Vos T, Flaxman AD, et al. A comparative risk assessment of burden of disease and injury attributable to 67 risk factors and risk factor clusters in 21 regions, 1990-2010: a systematic analysis for the global burden of disease study 2010. Lancet 2012;380:2224-60.

48 Ekoru K, Murphy GAV, Young EH, et al. Deriving an optimal threshold of waist circumference for detecting cardiometabolic risk in subSaharan Africa. Int J Obes 2018;42:487-94. 